\title{
ESTUDO DO PESO NEONATAL REGISTRADOS EM UM HOSPITAL E MATERNIDADE DA CIDADE DE SOBRAL/CE
}

\author{
STUDY OF NEONATAL WEIGHT IN A HOSPITAL AND MATERNITY \\ HOSPITAL IN THE CITY OF SOBRAL/CE
}

\begin{abstract}
Maria Carolina Quinderé de Almeida Frota ${ }^{1}$; Jéssica Sousa de Oliveira ${ }^{\mathbf{1}}$; Ana Roberta Gomes Severiano ${ }^{\mathbf{1}}$; Ana Rita Sampaio Carneiro $^{1}$; Antônio José de Miranda Dantas Terceiro ${ }^{1}$; Taíssa Braga da Silva ${ }^{1}$; José Klauber Roger Carneiro ${ }^{2}$; Maria Auxiliadora Silva Oliveira ${ }^{2}$

${ }^{1}$ Discentes do curso de Medicina do Centro Universitário Inta - UNINTA; Membro da Liga de Embriologia Integrada a Histologia - LAEH

${ }^{2}$ Docente do curso de Medicina do Centro Universitário Inta - UNINTA
\end{abstract}

\section{Resumo}

Objetivo: O peso ao nascer é um parâmetro usado, em todo o mundo, para avaliar as condições de saúde do recém-nascido. Com isso, o estudo objetivou analisar a associação entre o peso ao nascer e as variáveis maternas e obstétricas em um hospital do interior do estado do Ceará-Brasil. Metodologia: Foi realizado um estudo quantitativo e retrospectivo utilizando dados secundários a partir de prontuários de parturientes atendidas em um hospital e maternidade da cidade de Sobral, Estado do Ceará. Os dados analisados foram datados no ano de 2015. A pesquisa foi submetida e aprovada pelo Comitê de Ética da Universidade Estadual Vale do Acaraú - UVA. Resultados: No ano de 2015, foram preenchidas 1101 declarações de nascidos vivos residentes em Sobral, Estado do Ceará, sendo todas válidas como objeto de estudo. $\mathrm{O}$ peso de nascimento variou entre $2.500 \mathrm{~g}$ sendo considerado baixo peso (BPN) resultados menores que a média. A porcentagem de neonatos com BPN no período estudado foi de $27,2 \%$. Conclusão: Considerando o BPN um parâmetro perigoso e problemático no cenário atual, é importante que haja fortalecimento dos cuidados pré-natais e perinatais para prevenir e intervir, de forma eficaz, em resultados desfavoráveis para mãe e filho.

Palavras-chave: Baixo Peso; Peso ao Nascer; Recém-Nascido; Condições de Saúde do Neonato.

\begin{abstract}
Objectives: Birth weight is a parameter used throughout the world to evaluate the health status of the newborn. Thus, the study aimed to analyze the association between birth weight and maternal and obstetric variables in a hospital in the interior of the state of Ceará, Brazil. Methodology: A longitudinal and retrospective study was carried out using secondary data from records of parturients attended at a hospital and maternity hospital in the city of Sobral, State of Ceará. The data analyzed were dated in the year 2015. The research was submitted and approved by the Ethics Committee of Vale do Acaraú State University - UVA. Results: In the year 2015, 1101 declarations of live births resident in Sobral, State of Ceará, were completed, all of which are valid as object of study. The birth weight varied between 2,500 $\mathrm{g}$ and low weight (LBW) was considered lower than the mean. The percentage of neonates with LBW in the period studied was $27.2 \%$. Conclusion: Considering BPN a dangerous and problematic parameter in the current scenario, it is important that prenatal and perinatal care be strengthened to prevent and effectively intervene in unfavorable outcomes for mother and child.
\end{abstract}

Key words: Low Weight; Birth Weight; Newborn;

Neonate Health Conditions 


\section{INTRODUÇÃO}

O peso ao nascer é um parâmetro usado não apenas para indicar as condições intrauterinas em que a criança foi submetida durante o período gestacional (1), mas também avaliar a saúde do recémnascido e ser um dos principais determinantes para a sobrevivência do neonato (2). Por ser um indicador de saúde, seu déficit é considerado um fator de risco, principalmente, ao observar que existe uma relação diretamente proporcional entre o baixo peso ao nascer (BPN) e o grau de ocorrência de morbidade e mortalidade infantil, sendo considerado um dos principais fatores a determinar um risco significante para doenças e a probabilidade de sobreviver ao período neonatal e mesmo a todo o restante do primeiro ano de vida (3).

A criança que nasce com peso inferior a $2500 \mathrm{~g}$ é definida com BPN (4). Seus determinantes incluem, normalmente, a prematuridade ou a restrição de crescimento intrauterino (RCIU), ou, ainda, uma combinação de ambos (5). Normalmente, associa-se a baixos níveis de desenvolvimento socioeconômico, características maternas e de assistência maternoinfantil (6).

Os recém-nascidos considerados com baixo peso apresentam, principalmente alterações no desenvolvimento motor durante a gestação e lactação (7), mas também apresentam alterações do vínculo, diminuições do período de tempo de amamentação, maior risco de desenvolvimento de patologias crônicas durante a vida, uma maior propensão a atrasos de desenvolvimento e dificuldades de aprendizagem (8). Ademais, os neonatos com BPN podem apresentar maior predisposição para mortalidade nas primeiras semanas de vida, e, na vida adulta, apresentarão problemas mentais, orgânicos e neurológicos mais graves do que nas crianças nascidas com peso adequado (9).

Portanto, diante do exposto, as consequências do baixo peso ao nascer e suas repercussões caracteriza-o como um importante problema de saúde pública, além de ser um indicador global de saúde que traduz a eficiência do sistema de saúde local (10). Desse modo, conhecer o perfil do neonato a partir do peso, levando em consideração as variações, é importante, pois, pode expor fatores de risco relacionados. Sendo assim, o estudo objetivou analisar a associação entre o peso ao nascer e as variáveis maternas e obstétricas em um hospital do interior do estado do Ceará-Brasil.

\section{MÉTODOS}

Foi realizado um estudo quantitativo, descritivo e retrospectivo com análise documental utilizando dados secundários a partir de prontuários de parturientes atendidas em um hospital e maternidade da cidade de Sobral, estado do Ceará. Os dados analisados foram datados no ano de 2015.

Foram utilizadas informações significativas com o evento em estudo construídas a partir das seguintes variáveis contidas nos prontuários analisados: peso ao nascer, sendo considerado como BPN: $<2500$ $\mathrm{g}$ e peso normal e" 2500g; idade gestacional em semanas (de 22 a 27; de 28 a 36; de 37 a 41 ou e" 42); índice de Apgar (de 0 a 3; de 4 a 7 ou de 8 a 10); gênero (masculino; feminino); idade materna; número de consultas pré-natal (de 1 a 3 ; de 4 a 6 ou e" 7). Utilizou-se como critérios de exclusão dados não preenchidos das variáveis utilizadas.

Os resultados foram analisados em frequências absolutas e relativas, associando a variável dependente (peso ao nascer, contendo duas categorias: baixo peso e peso normal) com as demais variáveis estudadas.

A pesquisa foi submetida ao Comitê de Ética da Universidade Estadual Vale do Acaraú - UVA, tendo sido aprovada com número de parecer 1.402.425.

\section{RESULTADOS}

No ano de 2015 foram analisadas 1101 declarações de nascidos vivos (que corresponde a uma amostra de cerca de $50 \%$ do total) registrados no referido hospital, sendo todas válidas como objeto de estudo.

O peso de nascimento variou entre $<2500 \mathrm{~g}$ e e" $2500 \mathrm{~g}$ sendo considerado baixo peso (BPN) resultados menores que $2500 \mathrm{~g}$. A porcentagem de neonatos com BPN no período estudado foi de $27,2 \%$, enquanto os demais (801 neonatos em números absolutos) apresentaram o peso maior ou igual a $2.500 \mathrm{~g}$ (Tabela 01).

Do total de nascidos vivos, $51,4 \%$ foram do sexo masculino e $48,6 \%$ do sexo feminino. Contundo, percebe-se que o número de nascidos vivos com peso considerado ideal teve maior prevalência no sexo masculino (Tabela 02).

A variável idade gestacional apresentou associação bastante forte com o baixo peso ao nascer possibilitando importante prevalência de recém-nascido (RN) com baixo peso entre 28 a 36 semanas de gestação. Já os RN a termo (37 a 42 semanas) apresentaram-se em maior relevância no peso maior 
Tabela 01: Distribuição em número e proporção do peso ao nascer de neonatos em um hospital e maternidade de Sobral, 2015.

\begin{tabular}{ccccc}
\hline & $\leq 2.500 g$ & & \multicolumn{2}{c}{$\geq 2.500 g$} \\
\\
\hline $\mathbf{n}$ & & $\%$ & $\mathbf{n}$ & $\%$ \\
300 & 27,2 & 801 & 72,7 \\
\hline
\end{tabular}

Tabela 02: Número e proporção de nascidos vivos, segundo o gênero e o peso ao nascer em um hospital e maternidade de Sobral, 2015.

\begin{tabular}{ccccc}
\hline Sexo & $\leq \mathbf{2 . 5 0 0 g}$ & & \multicolumn{2}{c}{$\geq \mathbf{2 . 5 0 0 g}$} \\
& $\mathbf{n}$ & $\mathbf{\%}$ & $\mathbf{N}$ & $\%$ \\
\cline { 2 - 5 } & 145 & 25,8 & 415 & 74,2 \\
Feminino & 142 & 26,8 & 386 & 73,2 \\
\hline
\end{tabular}

Tabela 03: Número e proporção de nascidos vivos, segundo idade gestacional em um hospital e maternidade de Sobral, 2015.

\begin{tabular}{|c|c|c|c|c|}
\hline \multirow[t]{2}{*}{ Idade gestacional } & \multicolumn{2}{|c|}{$\leq 2.500 \mathrm{~g}$} & \multicolumn{2}{|c|}{$\geq 2.500 \mathrm{~g}$} \\
\hline & $\mathbf{n}$ & $\%$ & $\mathrm{n}$ & $\%$ \\
\hline De $22-27$ & 12 & 6,4 & -- & -- \\
\hline De $28-36$ & 114 & 61,2 & 53 & 8,9 \\
\hline De $37-41$ & 60 & 32,4 & 539 & 90,8 \\
\hline$\geq 42$ & & & 01 & 0,3 \\
\hline
\end{tabular}


Em relação ao índice de Apgar (método utilizado para avaliar o ajuste imediato do recém-nascido à vida extrauterina, interpretando suas condições de vitalidade) percebe-se que, em geral, a maioria dos recém nascidos tiveram notas de 8-10 relatando que os bebês nasceram em condições ótimas. No entanto, pode-se observar que há uma porcentagem maior de recém nascidos BPN com notas menores (entre 4-7 e 0-3) que a porcentagem de nascidos vivos com peso normal, relatando que há uma relação entre as condições de baixo peso ao nascer com dificuldades presentes na vida extrauterina ao primeiro minuto.

A idade materna variou de 12 a 54 anos e mostrou um impacto no peso do recém-nascido, visto que o segundo maior número de neonatos (em valor absoluto) com baixo peso ao nascer são filhos de mães entre 21 a 30 anos, comprovando a maior proporção de BPN em mães mais jovens, apresentando redução em idades intermediárias (Tabela 05).

Em relação ao número de consultas, que também apresentou associação direta com o baixo peso ao nascer, os resultados expressam a importância dos acompanhamentos pré-natais para um bom desenvolvimento do feto e seu conseguinte nascimento saudável, pois percebe-se que as mães que tiveram mais de 7 consultas pré-natais obtiveram também, em valores proporcionais, filhos com peso igual ou maior que 2.500 gramas (Tabela 06).

Tabela 04: Número e proporção de nascidos vivos, segundo o índice de Apgar e o peso. Sobral, 2015.

\begin{tabular}{|c|c|c|c|c|c|c|}
\hline \multirow{3}{*}{$\begin{array}{l}\text { Peso ao } \\
\text { nascer }\end{array}$} & \multicolumn{6}{|c|}{ Índice de Apgar 5' } \\
\hline & \multicolumn{2}{|c|}{$0-3$} & \multicolumn{2}{|c|}{$4-7$} & \multicolumn{2}{|c|}{$8-10$} \\
\hline & n & $\%$ & n & $\%$ & n & $\%$ \\
\hline$<2.500 \mathrm{~g}$ & 21 & 6,6 & 73 & 23,1 & 221 & 70,3 \\
\hline$\geq 2.500 \mathrm{~g}$ & 08 & 0,8 & 49 & 5,3 & 860 & 93,9 \\
\hline
\end{tabular}

Tabela 05: Número e proporção de nascidos vivos, segundo idade materna e peso em um hospital e maternidade de Sobral, 2015.

\begin{tabular}{rcccc} 
Idade & \multicolumn{2}{c}{$\leq \mathbf{2 . 5 0 0 g}$} & \multicolumn{2}{c}{$\geq \mathbf{2 . 5 0 0 g}$} \\
materna & $\mathbf{n}$ & $\mathbf{\%}$ & $\mathbf{n}$ & $\%$ \\
\cline { 2 - 5 } & 105 & 30,5 & 239 & 69,5 \\
De $\mathbf{1 2}-\mathbf{2 0}$ & 122 & 25,0 & 365 & 75,0 \\
De $\mathbf{2 1}-\mathbf{3 0}$ & 52 & 22,3 & 181 & 77,7 \\
De $\mathbf{4 1}-\mathbf{5 0}$ & 09 & 34,6 & 17 & 65,4 \\
De $\mathbf{5 1}-\mathbf{5 4}$ & 02 & 50,0 & 02 & 50,0
\end{tabular}


Tabela 06: Número e proporção de nascidos vivos, segundo o número de consultas e o peso em um hospital e maternidade de Sobral, 2015.
$\mathbf{N}^{\circ}$ de
$\leq \mathbf{2 . 5 0 0 g}$
$\geq 2.500 \mathrm{~g}$

consultas

\begin{tabular}{lcccc}
\hline & $\mathbf{n}$ & $\mathbf{\%}$ & $\mathbf{n}$ & $\mathbf{\%}$ \\
De 1-3 & 50 & 68,4 & 23 & 31,5 \\
De 4-6 & 111 & 38,4 & 178 & 61,5 \\
$\geq 7$ & 114 & 16,0 & 595 & 83,9 \\
\hline
\end{tabular}

\section{DISCUSSÃO}

Investigando os fatores associados ao baixo peso ao nascer no norte do Brasil, foi encontrado que $9,13 \%$ das crianças estudadas apresentava baixo peso ao nascer. No Brasil, a prevalência do baixo peso ao nascer tem permanecido estável, no patamar de $8 \%$, enquanto no mundo, este fator está presente em $15 \%$ de todos os nascimentos, trazendo consequências e complicações (11).

A prevalência de baixo peso ao nascer encontrada no presente trabalho foi de $27,4 \%$, uma taxa maior do que a apresentada pelo Brasil (8\%) e até mesmo, pelo mundo $(15,5 \%)$, refletindo as condições precárias encontradas nas condições de vida da mulher, bem como a qualidade da assistência recebida durante a gravidez, uma vez que, o baixo peso ao nascer é um importante indicador da saúde atual (12).

Sabendo que, nos países desenvolvidos, a ocorrência de BPN está envolvida, em sua maioria, com nascimentos pré-termo (13) e que, em crianças com baixo peso ao nascer, a prematuridade é um importante fator de risco para a mortalidade neonatal (14), evidencia-se a importância da longevidade a termo da gestação, visando as melhores condições para o neonato. Os resultados observados no estudo comprovam as citações acima, pois à medida que a duração da gestação aumenta (dificultando casos de prematuridade) a variável dependente também aumenta (Tabela 03).

Ademais, com associação entre a duração da gestação e a saúde do neonato percebe-se que à medida que a idade gestacional aumenta e que há ocorrências de partos a termo, aumenta as notas dos neonatos na escala de Apgar (15), ou seja, idade gestacional e índice de Apgar são variáveis interdependentes. Isto se confirma ao observar que neonatos BPN apresentam, em proporção, grandes dificuldades para alcançar notas entre 8 a 10 , enquanto obtém facilmente notas entre 0 a 3 (Tabela 04). No entanto, infere-se, de acordo com um estudo realizado na zona sul de São Paulo, que o RN prematuro pode nascer vigoroso e com Apgar adequado, mas manifestar agravamento de seu quadro, vindo a falecer posteriormente. Esse fato talvez explique porque a associação da mortalidade ocorre mais intensamente com o pequeno peso ao nascer, do que com as baixas pontuações do Apgar no $1^{\circ}$ minuto, agravando casos de associação do Apgar baixo e peso baixo com a mortalidade (16).

A associação entre a variável sexo e o BPN comprovam tendências observadas em outros estudos, como o realizado em Guaratinguetá, São Paulo, em que o sexo feminino é associado ao baixo peso ao nascer (17). Sendo assim, a prevalência de baixo peso foi maior nas crianças do sexo feminino $(26,8 \%)$ se comparadas com as do sexo masculino (25,8\%), visto na tabela 01 . A relação entre essas variáveis era esperada dado que para a mesma idade gestacional, as meninas apresentam menor peso que os meninos (5).

E da mesma forma como a tabela 01 desse artigo demonstra, um estudo feito no estado de Santa Catarina (18) também evidencia a prevalência de maior peso ao nascer para o sexo feminino, porém relacionando com a prematuridade dos neonatos. $\mathrm{O}$ 
estudo demonstrou que a prevalência de prematuridade no sexo masculino foi de $6,2 \%$, enquanto que entre as crianças do sexo feminino foi igual a 5,9\%.

Em relação a idade materna, vários estudos constatam uma incidência superior de BPN em grávidas tardias, considerando que a gravidez em mulheres com 35 anos ou mais está associada a um risco aumentado de aparecimento de complicações maternas (19). No entanto, na tabela 05 deste estudo evidencia-se que quanto menor a idade da gestante, maior os casos de neonatos abaixo de 2.500 gramas, comprovando-se essa afirmação com uma pesquisa realizada no Hospital Universitário da Universidade Federal do Maranhão (20), demonstrando também que as adolescentes, em geral, apresentaram proporções maiores de conceptos de BPN, correspondendo a 19,9\%, em relação às mulheres de 20 a 34 anos, na qual essa taxa foi de $14,2 \%$ diferença significante.

A relação de uma incidência superior de BPN em gestantes com menores idades pode ser explicada pela baixa adesão ao pré natal, uma vez que o número de consultas é outro importante variável associada ao BPN. Isso porque entre as gestantes adolescentes, provavelmente, existe uma maior possibilidade da realização de um pré-natal inadequado, já que esse fenômeno é muito mais presente em grupos sociais excluídos, desprovidas do apoio familiar, do companheiro e o amparo do Estado, com isso, a grávida adolescente inicia mais tardiamente o acompanhamento pré-natal e termina por fazer um menor número de consultas quando comparada às mulheres com vinte anos e mais (21).

Sabendo que o número de consultas é outro importante variável de associação com o BPN, evidenciou-se a importância das consultas para evitar resultados indesejados e melhorar a qualidade de vida do neonato e consequentemente da mãe. Considerando a realização de, no mínimo, seis consultas pré-natais como um indicador de acesso adequado ao serviço de saúde, a baixa cobertura pode refletir a dificuldade no acesso aos serviços de saúde e maior probabilidade de riscos à saúde da mãe e do neonato (22). Com isso, quando analisadas as categorias, observou-se que as mães que realizaram abaixo de 7 consultas obtiveram, em valores proporcionais, mais $\mathrm{RN}$ com baixo peso, comparadas às mães com mais de 7 consultas, corroborando que o número de consultas de prénatal associado a um adequado acompanhamento da gestação é abordado como a condição de intervenção para reduzir a incidência de complicações (23).

Uma pesquisa realizada em Pelotas, no Rio Grande do Sul (4), mostrou que há uma grande relação dos cuidados da gestante para a prevenção do baixo peso em neonatos, uma vez que, fatores como a depressão, desencadeiam uma nutrição defeituosa pela mãe e consequentemente para o feto, o que culmina altos riscos à mortalidade do feto ainda em espaço intrauterino, bem como ao nascer. $\mathrm{O}$ mesmo estudo ainda aponta os altos custos à saúde pública com internações e cuidados minuciosos e intensivos ao prematuro para que se restabeleça de um baixo peso. Logo, evidencia-se a importância do acompanhamento pré-natal durante as consultas abordado nesse referido artigo (Tabela 06).

\section{CONCLUSÃO}

O peso ao nascer é considerado um indicador de qualidade da saúde atual. Contudo, o baixo peso ao nascer, é considerado, atualmente, um problema de gestão na área da saúde, pois prediz riscos à saúde do neonato a curto prazo, como o de maior morbimortalidade, desnutrição no primeiro ano de vida, susceptibilidade a infecções, desconforto respiratório e traumas durante o parto.

Desse modo, a prevalência do baixo peso ao nascer observada neste estudo demonstra uma falta de assistência adequada quando comparadas a estudos de outros estados ou municípios. Os fatores associados ao BPN são frequentemente idade materna, idade gestacional e número de consultas. Assim, propõe-se melhoria na qualidade, não apenas da assistência pré-natal, como também da assistência ginecológico-obstétrica, com o objetivo de prevenir gravidez precoce assim como instruir sobre os cuidados e deveres necessários das mães para com os seus filhos, começando pela tentativa de aumento no número de consultas prénatais, o que possibilitaria melhores condições de vida para o binômio mãe e filho e a redução nos custos para a saúde pública.

Sendo assim, considerando o BPN um parâmetro perigoso e problemático no cenário atual, é importante que haja fortalecimento dos cuidados pré-natais e perinatais para prevenir e intervir, de forma eficaz, em resultados desfavoráveis para mãe e filho. 


\section{REFERÊNCIAS}

1. Viana KJ, Taddei JAAC, Cocetti M, Warkentin S,. Peso ao nascer das crianças brasileiras menores de dois anos. Cad. Saúde Pública, Rio de Janeiro, 29(2):349-356, fev, 2013. Disponível: http://www.scielo.br/pdf/csp/v29n2/21.pdf

2. Tourinho AB, Reis LBSM. Peso ao nascer: Uma abordagem nutricional. Programa de Residência em Nutrição Clínica, Hospital Regional da Asa Norte, Secretaria de Estado de Saúde do Distrito Federal, 2012; agosto; 20. Disponível: http:// bvsms.saude.gov.br/bvs/periodicos/revista_ESCS_v23_n1_a02_peso_ao_nascer.pdf

3. Santos SP, Oliveira LMB. Baixo peso ao nascer e sua relação com obesidade e síndrome metabólica na infância e adolescência. Artigo de revisão. Revista de Ciências Médicas e Biológicas; Bahia, BA, Brasil, 2010. Disponível: http:// www.repositorio.ufba.br:8080/ri/bitstream/ri/5723/1/5898-16360-1-PB\%5B1\%5D.pdf

4. Menezes LO, Pinheiro RT, Quevedo LA, Oliveira SS, Silva RA; Pinheiro KAT, Santo GCE, Jansen K. O impacto do baixo peso ao nascer relacionado à depressão gestacional para o financiamento federal da saúde pública: uma análise do Município de Pelotas, Rio Grande do Sul, Brasil. Universidade Católica de Pelotas. Aprovado em 13/Jul/2012. Disponível: http://www.scielo.br/scielo.php?script=sci_arttext\&pid=S0102-311X2012001000012

5. Maia RRP, Souza JMP. Fatores associados ao baixo peso ao nascer em município do norte do Brasil. Revista Bras. Crescimento Desenvolvimento Hum. 2010; 20(3) 735-744. Disponível: http://pepsic.bvsalud.org/pdf/rbcdh/v20n3/08.pdf 6. Araújo LMCRS. Factores associados com o Baixo Peso ao Nascer. Dissertação de mestrado. Instituto Politécnico de Viseu (ESEV), Portugal; 2013; 23-28. Disponível: http://repositorio.ipv.pt/bitstream/10400.19/1735/1/

ARAUJO\%20Lucia\%20Margarida\%20Correia\%20Reis\%20Sousa\%20-\%20Disserta\%C3\%A7\%C3\%A3o\%20mestrado.pdf 7. Santos DCC, Campos D, Gonçalves VMG, Mello BBA, Campos TM, Gagliardo HGRG. Influência do baixo peso ao nascer sobre o desempenho motor de lactentes a termo no primeiro semestre de vida. Rev. bras. fisioter. Vol. 8, No. 3 (2004), 261266. Disponível: https://www.researchgate.net/profile/Denise_Santos2/publication/

228691237_Influencia_do_Baixo_peso_ao_nascer_sobre_o_desempenho_motor_de_lactentes_a_termo_no_primeiro_semestre_de_vida/ links/00b7d529be6465f55b000000.pdf

8. Coutinho E, Araújo L, Pereira C, Duarte J, Nelas P, Chaves C. Fatores associados ao baixo peso ao nascer. Revista Infad de Psicología, vol. 1; n. 2 (2016). Disponível: http://www.infad.eu/RevistaINFAD/OJS/index.php/IJODAEP/article/view/229/514 9. Capelli JCS, Pontes JS, Pereira SEA, Silva AAM, Carmo CN, Boccolini CS, Almeida MFL. Peso ao nascer e fatores associados ao período pré-natal: um estudo transversal em hospital maternidade de referência. Ciênc. saúde coletiva [online]. 2014, vol.19, n.7, pp.2063-2072. ISSN 1413-8123. Disponível: http://dx.doi.org/10.1590/1413-81232014197.20692013. 10. Minamisawa R, Barbosa MA, Malagoni L, Andraus, LMS. Fatores associados ao baixo peso ao nascer no Estado de Goiás. Revista Eletrônica de Enfermagem, v. 06, n. 03, p. 336-349, 2004. Disponível: https://www.fen.ufg.br/fen_revista/ revista6_3/pdf/04_Original.pdf

11. Alves TL, Ribeiro Júnior HC, Costa ML, Valois SS. Fatores associados ao recém-nascido pequeno para a idade gestacional: uma revisão. Artigo de revisão. Nutrire. 2015 Dec;40(3):376-382. Recebido: Set 03, 2013 Aprovado: Jul. 22, 2015. Disponível: http://sban.cloudpainel.com.br/files/revistas_publicacoes/485.pdf

12. Valois AA. Fatores associados ao ganho de peso neonatal em prematuros de muito baixo peso ao nascer. Dissertação de Pós Graduação. Universidade Federal de Pernambuco (UFPE); Centro de Ciências da Saúde; Programa de Pós Graduação em Saúde da Criança e do Adolescente. Recife, PE, Brasil. 2013; 15-34. Disponível: http://repositorio.ufpe.br/ bitstream/handle/123456789/13253/Disserta\%C3\%A7\%C3\%A3o\%20Amanda\%20Valois.pdf? sequence=1\&isAllowed=y 13. Caçola P, Bobbio TG. Baixo peso ao nascer e alterações no desenvolvimento motor: a realidade atual. Revista Paulista Pediatria 2010;28(1):70-6. São Paulo, SP, Brasil. Disponível: http://www.spsp.org.br/spsp_2008/revista/RPP_v28n1_p70-6.pdf 14. Gaiva MAM, Fujimori E, Sato APS. Mortalidade neonatal em crianças com baixo peso ao nascer. Rev Esc Enferm USP 2014; 48(5):778-86. Disponível: http://www.scielo.br/pdf/reeusp/v48n5/pt_0080-6234-reeusp-48-05-778.pdf 15. Figueiró-Filho EA, Oliveira VM, Ferreira CM, Silva VM, TINOS, Silva AL, Kanomata LB. Variáveis perinatais e associação de recém-nascidos de muito baixo peso ao nascer em hospital público universitário do Brasil. Rev Bras Ginecol Obstet. 2014; 36(1):10-6. Campo Grande (MG), Brasil, 2014. Disponível: http://www.scielo.br/pdf/rbgo/v36n1/0100-7203rbgo-36-01-00010.pdf

16. Oliveira TG, Freire PV, Moreira FT, Moraes JSB, Arrelaro RCA, Rossi S et al. Escore de Apgar e mortalidade neonatal em um hospital localizado na zona sul do município de São Paulo. Einstein. 2012;10(1):22-8. Disponível: http://www.scielo.br/ pdf/eins/v10n1/pt_v10n1a06.pdf

17. Uchimura TT, Pelissari DM, Uchimura NS. Baixo peso ao nascer e fatores associados. Revista Gaúcha Enfermagem, Porto Alegre (RS), 2008, mar; 29: 33-8. Disponível: http://www.seer.ufrgs.br/RevistaGauchadeEnfermagem/article/viewFile/ $5261 / 2995$

18. Franciotti DL, Mayer GN, Cancelier ACL. Fatores de risco para baixo peso ao nascer: um estudo de caso-controle. Arquivos Catarinenses de Medicina Vol. 39, nº 3, de 2010. Santa Catarina, SC, Brasil. Disponível: http://www.acm.org.br/ revista/pdf/artigos/818.pdf 
19. Nogueira JMP. Factores associados ao Baixo Peso à Nascença. Faculdade de Ciências da Nutrição e Alimentação, Universidade do Porto. Portugual, 2010. Disponível: https://repositorio-aberto.up.pt/bitstream/10216/54473/3/

139111_1058TCD58.pdf

20. Santos GHN, Martins MG, Sousa MS. Gravidez na adolescência e fatores associados com baixo peso ao nascer. Rev Bras Ginecol Obstet. 2008; 30(5): 224-31. Disponível: http://www.scielo.br/pdf/rbgo/v30n5/a04v30n5

21. Lages CDR, SOUSA, Oliveira JC, Cunha KJB, Silva NC, Santos TMMG. Fatores preditores para a admissão do recémnascido na unidade de terapia intensiva. Submetido: 24/07/2013; Aceito: 13/01/2014. Rev Rene. 2014 jan-fev; 15(1):3-11.

Disponível: file:///C:/Users/Brenda/Downloads/3068-5726-1-SM.pdf

22. Silva SS, Santos FDD, Coca LL. Nascimento de recém-nascidos de baixo PESO em instituição filantrópica terciária do Município de Piracicaba. Revista Electrónica Trimestral de Enfermería, Enfermeria Global. No 23 Julio 2011. Portugal. Disponível: http://scielo.isciii.es/pdf/eg/v10n23/pt_clinica6.pdf

23. Sass A, Gravena AAF, Pelloso SM, Marcon SS. Resultados perinatais nos extremos da vida reprodutiva e fatores associados ao baixo peso ao nascer. Rev Gaúcha Enferm., Porto Alegre (RS) 2011 jun;32(2):352-8. Disponível: http:// www.seer.ufrgs.br/RevistaGauchadeEnfermagem/article/viewFile/14528/13059 